\title{
Loss of tumour-specific ATM protein expression is an independent prognostic factor in early resected NSCLC
}

\author{
Lars F. Petersen ${ }^{1}$, Alexander C. Klimowicz ${ }^{2,7}$, Shannon Otsuka ${ }^{1}$, Anifat A. Elegbede ${ }^{1}$, \\ Stephanie K. Petrillo', Tyler Williamson ${ }^{3}$, Chris T. Williamson ${ }^{4}$, Mie Konno ${ }^{2}$, Susan P. \\ Lees-Miller ${ }^{5}$, Desiree Hao ${ }^{1}$, Don Morris ${ }^{1,2}$, Anthony M. Magliocco ${ }^{6}$, D. Gwyn Bebb ${ }^{1}$ \\ ${ }^{1}$ Department of Oncology, Tom Baker Cancer Centre and University of Calgary, Calgary, Alberta, T2N 4N2, Canada \\ ${ }^{2}$ Functional Tissue Imaging Unit, Translational Research Laboratory, Tom Baker Cancer Centre, Calgary, Alberta, T2N 4N2, Canada \\ ${ }^{3}$ Department of Community Health Sciences, TRW Building, University of Calgary, Calgary, Alberta, T2N 4Z6, Canada \\ ${ }^{4}$ Gene Function Lab, The Institute for Cancer Research, London, SW3 6JB, UK \\ ${ }^{5}$ Department of Biochemistry and Molecular Biology, HRIC Building, University of Calgary, Calgary, Alberta, T2N 4Z6, Canada \\ ${ }^{6}$ Department of Anatomic Pathology, H. Lee Moffitt Cancer Center, Tampa, FL, 33612, USA \\ ${ }^{7}$ Present Address: Immunology and Inflammation Research, Boehringer Ingelheim Pharmaceuticals, Inc., Ridgefield, CT, 06877, USA \\ Correspondence to: D. Gwyn Bebb, email: Gwyn.Bebb@albertahealthservices.ca \\ Keywords: ATM, non-small cell lung cancer, outcome, early stage, digital pathology
}

Received: October 06, 2016

Accepted: February 21, 2017

Published: March 15, 2017

Copyright: Lars F. Petersen et al. This is an open-access article distributed under the terms of the Creative Commons Attribution License 3.0 (CC BY 3.0), which permits unrestricted use, distribution, and reproduction in any medium, provided the original author and source are credited.

\section{ABSTRACT}

Ataxia-telangiectasia mutated (ATM) is critical in maintaining genomic integrity. In response to DNA double-strand breaks, ATM phosphorylates downstream proteins involved in cell-cycle checkpoint arrest, DNA repair, and apoptosis. Here we investigate the frequency, and influence of ATM deficiency on outcome, in early-resected nonsmall cell lung cancer (NSCLC). Tissue microarrays, containing 165 formalin-fixed, paraffin-embedded resected NSCLC tumours from patients diagnosed at the Tom Baker Cancer Centre, Calgary, Canada, between 2003 and 2006, were analyzed for ATM expression using quantitative fluorescence immunohistochemistry. Both malignant cellspecific ATM expression and the ratio of ATM expression within malignant tumour cells compared to that in the surrounding tumour stroma, defined as the ATM expression index (ATM-EI), were measured and correlated with clinical outcome. ATM loss was identified in $\mathbf{2 1 . 8} \%$ of patients, and was unaffected by clinical pathological variables. Patients with low ATM-EI tumours had worse survival outcomes compared to those with high ATM-EI $(p<0.01)$. This effect was pronounced in stage II/III patients, even after adjusting for other clinical co-variates $(p<0.001)$. Additionally, we provide evidence that ATM-deficient patients may derive greater benefit from guideline-recommended adjuvant chemotherapy following surgical resection. Taken together, these results indicate that ATM loss seems to be an early event in NSCLC carcinogenesis and is an independent prognostic factor associated with worse survival in stage II/III patients.

\section{INTRODUCTION}

Recent studies have suggested that non-small cell lung cancer (NSCLC) exhibits a greater degree of genetic instability than most other malignancies $[1,2]$. The cause of such genomic instability in NSCLC is not well understood. Although associated with extensive mutagen exposure through smoking in $80 \%$ of patients, this genetic instability is surprisingly matched and even exceeded in non-smoking related cases [3]. Genetic heterogeneity, both between and within tumours, has been proposed as a barrier to successful treatment [4] of the advanced disease stage with which most NSCLC patients present, contributing to the disappointing $15 \%$ overall five-year survival [5]. While substantial heterogeneity in both the degree and character of such genomic instability has been described [6], it is postulated that dysregulation of the DNA damage response (DDR) is a common means by which such a "mutator phenotype" $[7,8]$ is generated. 
ATM (ataxia-telangiectasia mutated) plays a key role in the cellular DNA damage response (DDR) [9-11], and is emerging as an important tumour-suppressor biomarker in many malignancies, as supported by data suggesting that $15 \%$ of gastric cancers carry ATM mutations $[12,13]$ and that ATM deficiency is prevalent in lymphoid malignancies $[14,15]$. Furthermore, in an analysis of listed mutations in 500 human protein kinases in 169 primary tumours and 40 cell lines, mutations in ATM emerged at number 3 in terms of frequency. A large proportion of these mutations were found in lung cancers, which exhibited the highest rate of somatic ATM mutations of all tumours analyzed [16]. Several other studies have confirmed that NSCLC harbors DNA repair deficiencies due to ATM mutations [17]. In two recently published studies, the frequency of ATM deficiency in lung adenocarcinomas was investigated and shown to range from $18 \%$ to as high as $40 \%[18,19]$. The impact on ATM loss was not associated with overall survival [19]. In this particular study the authors assessed total global ATM in patient samples using standard histopathalogical immunohistochemistry (IHC) staining. While clinically valid, this method is not quantitative, nor does it account for tissue heterogeneity within the sample.

To address this, we set out to determine the frequency and clinical significance of loss of ATM expression in a series of early stage, resected cases of NSCLC using quantitative fluorescence IHC. We expect that somatic changes in ATM would lead to a loss of ATM expression that could be detected by quantifying the relative expression of ATM protein within the malignant versus the stromal component of the tumour - a concept introduced here as the ATM expression index (ATM-EI). The ATM-EI should be a more stringent measure of ATM loss in tumours, and we hypothesized that increased genomic instability associated with ATM loss [20] in the early stages of NSCLC and would be associated with a worse clinical outcome. Here, we demonstrate that approximately $20 \%$ of NSCLC cases show a reduced ATM expression in the malignant compared to the nonmalignant components of the tumour and that this loss of ATM expression is associated with a worse prognosis. Furthermore, we present data suggesting that patients with low ATM-EI respond more positively to adjuvant chemotherapy. Together, our data shows that tumour ATM loss has prognostic significance in early stage NSCLC, and could identify a unique cohort of patients who can benefit from disease-modifying therapy.

\section{RESULTS}

\section{Patient characteristics}

To investigate the clinical significance of ATM expression in NSCLC, we measured ATM protein expression in resected tumours. Our investigation adheres to the REMARK criteria for the study of biomarkers [21]. Of 1507 diagnoses of NSCLC between January 2003 and December 2006, 165 patients underwent full resection for stage I, II, or IIIA disease. The majority was classified as stage I $(n=104)$, with 45 stage II and 16 stage III patients. Demographic characteristics and survival were compatible with historical controls. Patient demographics and clinical characteristics are shown in Table 1. Briefly, mean age was 64 years, $53.3 \%$ were female, $87.9 \%$ were current or ex-smokers, and $6.7 \%$ were of Southeast Asian ethnicity; $53.9 \%$ were adenocarcinomas, $28.5 \%$ squamous cell carcinomas, and $17.6 \%$ had other tumour histology (which included bronchoalveolar carcinoma, large cell carcinoma, adenosquamous carcinoma and sarcomatid carcinoma). The majority of the patients (67.2\%) underwent a lobectomy as their resection type, and $40.6 \%$ (34 stage IB, 11 IIA, 10 IIB, 12 IIIA) received adjuvant treatment. At the end of study date (November 30,2010), 57.6\% of the patients were still alive.

\section{ATM expression analysis and determination of ATM deficiency}

ATM protein expression levels were measured by quantitative fluorescence immunohistochemistry using the HistoRx AQUA ${ }^{\circledR}$ platform [22] (Figure 1). Antibody specificity was validated using the L3 ATM-deficient human lymphoblastoid cell line and the BT/C3ABR age matched ATM-proficient human lymphoblastoid cell line as controls (Figure 1A, left panels) [23]. ATM expression in normal lung tissue demonstrated a relatively uniform and intense nuclear expression pattern in both epithelial (pan-cytokeratin positive) and non-epithelial cell types (vimentin positive and pan-cytokeratin negative), consistent with the reported cellular distribution of ATM (Figure 1B). In contrast, ATM expression in lowexpressing NSCLC samples had only faint and/or sporadic nuclear staining pattern in malignant cells compared with a stronger distinct nuclear staining pattern in adjacent stromal cells (Figure 1B). High ATM-expressing tumours, similar to normal lung tissue, had a strong distinct nuclear expression pattern in both malignant epithelial and stromal cells (Figure 1B).

To determine whether ATM expression was altered in NSCLC compared to normal lung epithelium we compared ATM expression within our NSCLC patient cohort to a $95 \%$ confidence interval (C.I.) around the median results from normal lung tissue (Figure 1C and 1D). Malignant cell-specific ATM protein expression was calculated within each patient tissue core as the average ATM pixel intensity within the pancytokeratin positive malignant cell area. Median ATM expression in normal lung epithelium was 3531 (95\% C.I.: 1931-5130) (Figure 1C, solid and hashed blue lines). Median ATM expression in our NSCLC cohort was 4490 (Figure 1C, red line) and fell in the upper end of 
Table 1: Demographic characteristics of early stage lung cancer cohort - comparing low and high ATM expression

\begin{tabular}{|c|c|c|c|c|c|c|}
\hline & \multicolumn{6}{|c|}{ Number of Patientsa (\%) } \\
\hline & Characteristic & $\begin{array}{l}\text { Full Cohort } \\
\quad(n=165)\end{array}$ & $\begin{array}{c}\text { Low Malignant } \\
\text { Cell ATM }(n=77)\end{array}$ & $\begin{array}{c}\text { High Malignant Cell } \\
\operatorname{ATM}(n=88)\end{array}$ & $\begin{array}{c}\text { Low } \\
\text { ATM-EI } \\
(n=36)\end{array}$ & $\begin{array}{c}\text { High } \\
\text { ATM-EI } \\
(n=129)\end{array}$ \\
\hline Age & Mean (SD) & $64.1(9.8)$ & $64.5(10.4)$ & $63.7(9.3)$ & $63.5(11.1)$ & $64.3(9.4)$ \\
\hline \multirow[t]{2}{*}{ Gender } & Male & $77(46.7)$ & $41(53.3)$ & $36(40.9)$ & $17(47.2)$ & $60(46.5)$ \\
\hline & Female & $88(53.3)$ & $36(46.8)$ & $52(59.1)$ & $19(52.8)$ & $69(53.5)$ \\
\hline \multirow[t]{2}{*}{ Ethnicity } & South East Asian & $11(6.7)$ & $6(7.8)$ & $5(5.7)$ & $2(5.6)$ & $9(7.0)$ \\
\hline & Other & $154(93.3)$ & $71(92.2)$ & $83(94.3)$ & $34(94.4)$ & $120(93.0)$ \\
\hline \multirow{2}{*}{ Smoking Status } & Ever $^{b}$ & $145(87.9)$ & $68(88.3)$ & $77(87.5)$ & $34(94.4)$ & $111(86.1)$ \\
\hline & Never & $20(12.1)$ & $9(11.7)$ & $11(12.5)$ & $2(5.6)$ & $18(14.0)$ \\
\hline \multirow[t]{4}{*}{ Histology } & Adenocarcinoma & $89(53.9)$ & $43(55.8)$ & $46(52.3)$ & $25(69.4)$ & $64(49.6)$ \\
\hline & Squamous Cell & $47(28.5)$ & $22(28.6)$ & $25(28.4)$ & $6(16.7)$ & $41(31.8)$ \\
\hline & $\mathrm{BAC}$ & $18(10.9)$ & $8(10.4)$ & $10(11.4)$ & $2(5.6)$ & $16(12.4)$ \\
\hline & Other $^{\mathrm{c}}$ & $11(6.7)$ & $4(5.2)$ & $7(8.0)$ & $3(8.3)$ & $8(6.2)$ \\
\hline \multirow[t]{2}{*}{ Stage } & I & $104(63.0)$ & $48(62.3)$ & $56(63.6)$ & $24(66.7)$ & $80(62.0)$ \\
\hline & II/III & $61(37.0)$ & $29(37.7)$ & $32(36.4)$ & $12(33.3)$ & $49(38.0)$ \\
\hline \multirow{3}{*}{$\begin{array}{l}\text { Surgical } \\
\text { Method }\end{array}$} & Lobectomy & $110(66.7)$ & $53(68.8)$ & $57(64.8)$ & $28(77.8)$ & $82(63.6)$ \\
\hline & Wedge Resection & $35(21.2)$ & $13(16.9)$ & $22(25.0)$ & $5(13.9)$ & $30(23.2)$ \\
\hline & Pneumonectomy & $20(12.1)$ & $11(14.3)$ & $9(10.2)$ & $3(8.3)$ & $17(13.2)$ \\
\hline Perioperative & Yes & $67(40.6)$ & $30(39.0)$ & $37(42.0)$ & $10(27.8)$ & $57(44.2)$ \\
\hline Chemotherapy & No & $98(59.4)$ & $47(61.0)$ & $51(58.0)$ & $26(72.2)$ & $72(55.8)$ \\
\hline
\end{tabular}

Note: $\mathrm{SD}=$ standard deviation.

${ }^{a}$ unless otherwise stated.

bincludes current, ex, unknown (unknown =2).

'other includes adenosquamous, large cell, sarcomatoid.

Column totals may not add to one hundred due to rounding.

There were no statistically significant differences in characteristics between low and high ATM.

the normal lung epithelium 95\% C.I., indicating moderate overexpression in tumour tissue.

The ATM-EI was defined as the expression of ATM within the malignant cells of the tumour relative to the ATM expression in the adjacent tumour-associated nonmalignant stromal cells. This ATM expression index was calculated by dividing average ATM pixel intensity within the pan-cytokeratin-positive malignant cell area by the average ATM pixel intensity within the corresponding vimentin-positive and pan-cytokeratin-negative tumourassociated stromal area. We hypothesized that the ATM-EI would be a more meaningful measure of ATM deficiency, as it would take into account the heterogeneity of normal ATM expression levels between patients. A low ATM-EI represents a tumour sample in which the tumour portion is ATM deficient relative to the surrounding stromal cells. The median ATM-EI in normal lung epithelium was 0.906 (95\% C.I.: 0.747-1.065) (Figure 1D, solid and hashed blue lines). The median ATM expression index in our NSCLC cohort was 0.851 (Figure 1D, red line) and fell in the lower end of the normal lung epithelium ATM-EI 95\% C.I., indicating modest under expression in tumour tissue.

\section{Influence of ATM deficiency on outcome}

Patients were dichotomized by malignant cell-specific ATM expression and the ATM-EI for survival analysis using the Kaplan-Meier method. Patients were separated into those with a high ATM score and those with a low ATM score using a cut-point based on the log rank statistic [24] (Figure 2). This method identified a malignant cell-specific ATM expression cut-point of 4399, defining 77/165 (46.7\%) of patients as being ATM-deficient (Table 1). In contrast, the ATM-EI cut point of 0.716 defined 36/165 (21.8\%) of patients as being ATM-deficient (Table 1). There were no significant differences in the clinicopathological features between the high- and low-ATM expressing patients using either method to define the groups (Table 1).

Compared to patients with a high malignant cell-specific ATM expression, patients with a low ATM 
expression had worse 5-year disease free survival (DFS) and 5-year overall survival (OS) (Figures 2A and 2B). Similarly, compared with patients that had a high ATM-EI, patients with a low ATM-EI had significantly worse DFS and OS (Figures 2C and 2D). Further analysis showed that the effect of ATM expression levels on DFS and OS is different depending on stage. Among stage I patients, there was no
ATM expression effect with either malignant cell-specific ATM or ATM-EI measurements on DFS or OS. However, for stage II/III patients, there is a significant effect on both DFS and OS, using both ATM measurements (Figure 3). As with the overall cohort analysis in Figure 3, the associations between the ATM-EI and survival are stronger than those between malignant cell-specific ATM expression and survival.
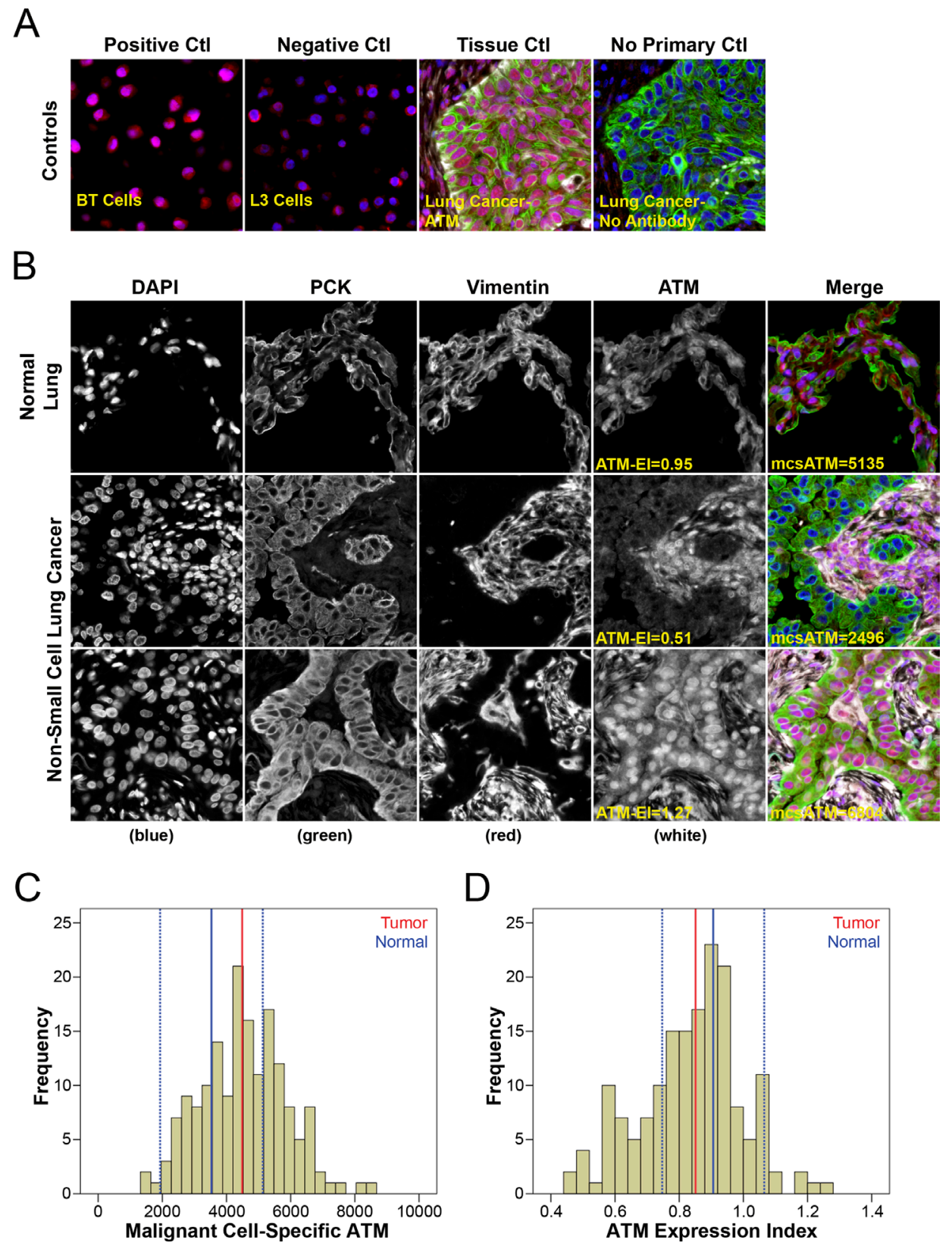

Figure 1: Quantitative fluorescence immunohistochemistry and digital image analysis for ATM in NSCLC. (A) Control ATM antibody specificity staining in ATM positive BT cells and ATM deficient L3 cells, as well as in lung cancer tissue with and without primary anti-ATM antibody. (B) Representative fluorescence images for ATM expression in normal lung tissue and NSCLC. Both ATM expression index (ATM-EI) and malignant cell-specific ATM (mcsATM) scores are reported. DAPI-stained nuclei are depicted in blue, pan-cytokeratin-stained epithelial/malignant cells are depicted in green, vimentin-stained non-malignant tumour stromal cells are depicted in white, and ATM protein expression is depicted in red. Histogram distributions representing malignant cell-specific ATM expression (C) and the ATM expression index (D) within the NSCLC cohort. The solid blue line represents median ATM expression in normal lung tissue, the broken blue lines represent $+/-2$ standard deviations from median ATM expression in normal lung tissue, and the solid red line represent median ATM expression in NSCLC. 
Cox proportional hazards models were used to determine if ATM loss identified by either malignant cellspecific ATM expression or the ATM-EI was an independent prognostic factor for DFS or OS. A stage specific approach was adopted for this analysis as stage was shown to modify the ATM survival relationship in the univariate analysis. Neither malignant cell-specific ATM expression nor the ATM-EI demonstrated a prognostic effect for patients with stage I disease (data not shown). However, after adjusting for histology, gender, age, and adjuvant treatment, there was a significant association between the ATM-EI and DFS (HR: 4.75, 95\% CI: 2.02 to $11.17, p<0.001$ ) among those with stage II/III disease (Table 2). When examining OS, and after adjusting for the same factors, the ATM-EI was again a significant prognostic factor (HR: 5.09, 95\%
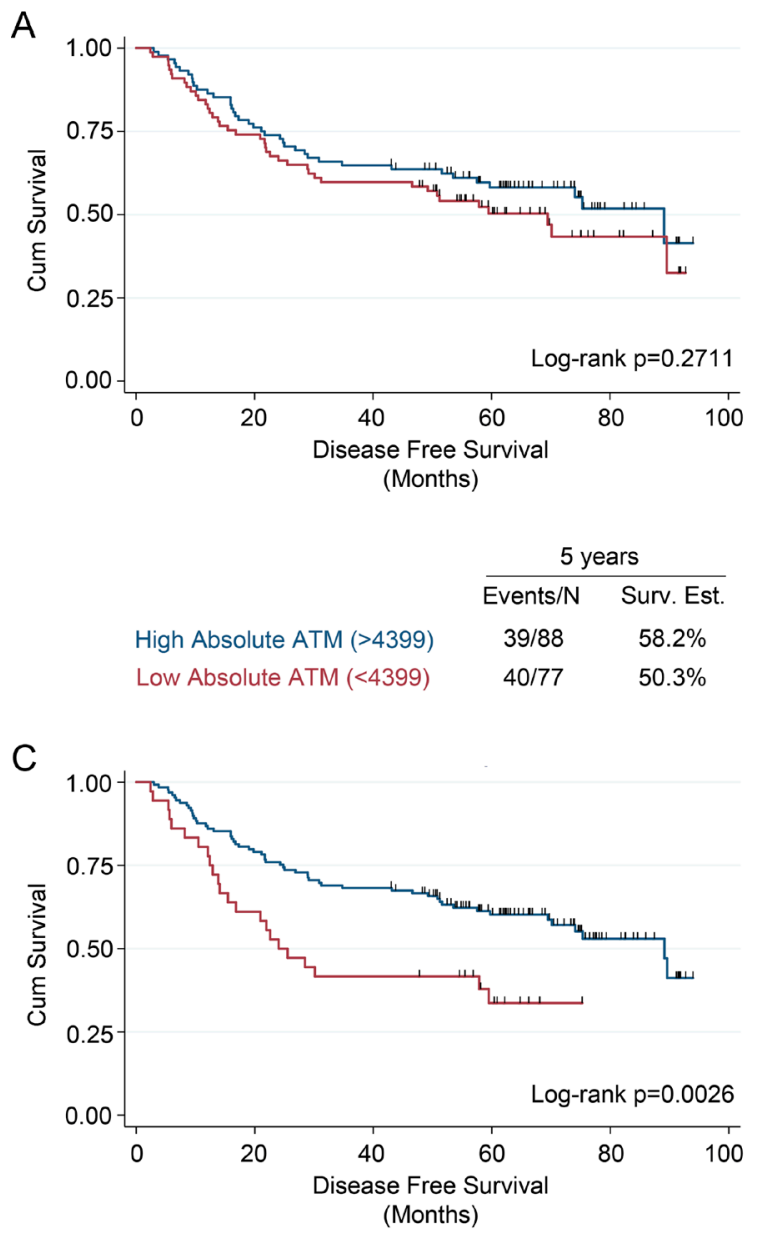

\begin{tabular}{lcc} 
& \multicolumn{2}{c}{5 years } \\
\cline { 2 - 3 } & Events/N & Surv. Est. \\
High Relative ATM $(>0.716)$ & $56 / 129$ & $60.2 \%$ \\
Low Relative ATM $(<0.716)$ & $23 / 36$ & $33.7 \%$
\end{tabular}

CI: 2.07 to $12.52, p<0.001)$ as well as malignant cellspecific ATM (HR: $2.79,95 \%$ CI: 1.38 to $5.66, p=0.004$ ) among those with stage II/III disease (Table 2). Additional analyses explored whether or not the prognostic effect of the ATM-EI was being driven by the stage III patients $(n=16)$. No difference in the ATM effect was observed between the stage II and the stage III patients (DFS $\mathrm{HR}=2.07,95 \% \mathrm{CI}: 0.32$ to $13.48, p=0.45$; $\mathrm{OS}-\mathrm{HR}=$ $1.15,95 \% \mathrm{CI}: 0.18$ to $7.24, p=0.88$ ).

\section{ATM deficient patients respond to perioperative chemotherapy}

To assess the therapeutic impact of low ATM EI in this setting we compared outcomes among those
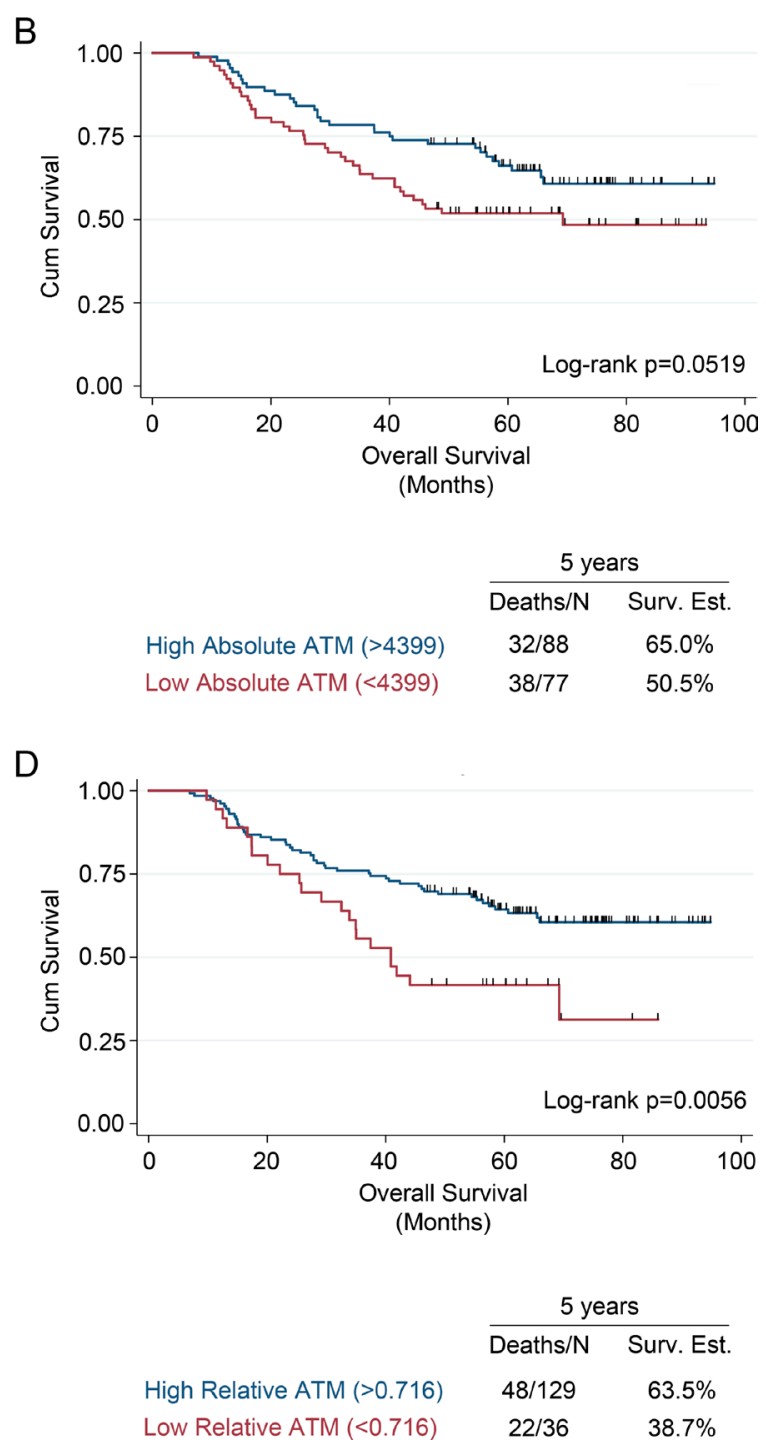

Figure 2: Univariate survival analysis in early stage NSCLC patients based on ATM expression measured using malignant cell-specific ATM and ATM expression index scores. Kaplan-Meier analysis and 5-year survival estimates for (A) disease free survival and (B) overall survival in NSCLC patients with low or high malignant cell specific ATM expression scores. Kaplan-Meier analysis and 5-year survival estimates for (C) disease free survival and (D) overall survival in NSCLC patients with low or high ATM expression index scores. All Kaplan-Meier curves include the 5-year event rate expressed at the Events/N, where $\mathrm{N}$ is the number at risk. 
patients who received perioperative systemic therapy. Periopertative chemotherapy (platin/vinorelbine combination chemotherapy) was given to $40.6 \%(67 / 165)$ of patients in our cohort of whom 64 received adjuvant chemo, 1 received neoadjuvant chemo, and 1 received neoadjuvant chemo/ radiation therapy. When sorted into high and low ATMEI groups, patients with low ATM-EI treated with platin based perioperative treatment showed a strong trend toward improved disease free survival, $(10 / 36 ; p=0.052)$, while there was virtually no difference in DFS among treated or untreated patients with high ATM-EI $(57 / 129 ; p=0.965)$.

A
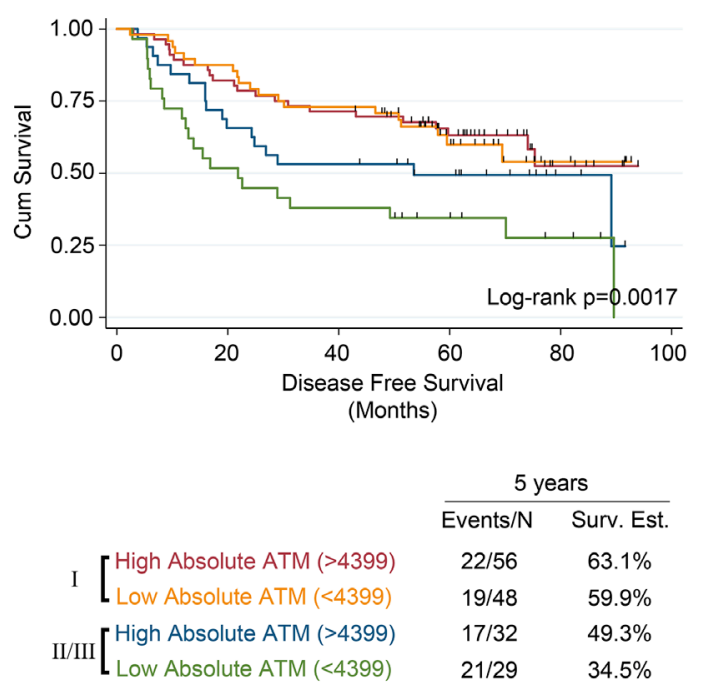

C
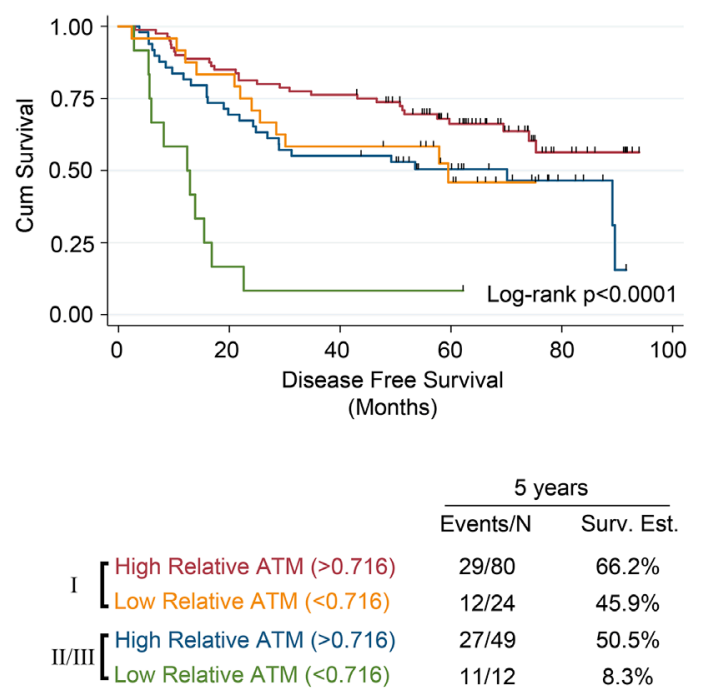

This suggests that low ATM EI may be predictive of benefit from adjuvant platin based treatment. (Figure 4).

\section{DISCUSSION}

The key role held by ATM in maintaining genomic integrity as part of the DDR suggests that early loss of ATM function in the process of malignant transformation could herald the generation of a mutator phenotype, an "enabling feature" in the evolution of cancer [25]. Our findings on clinical outcome in patients diagnosed with

B
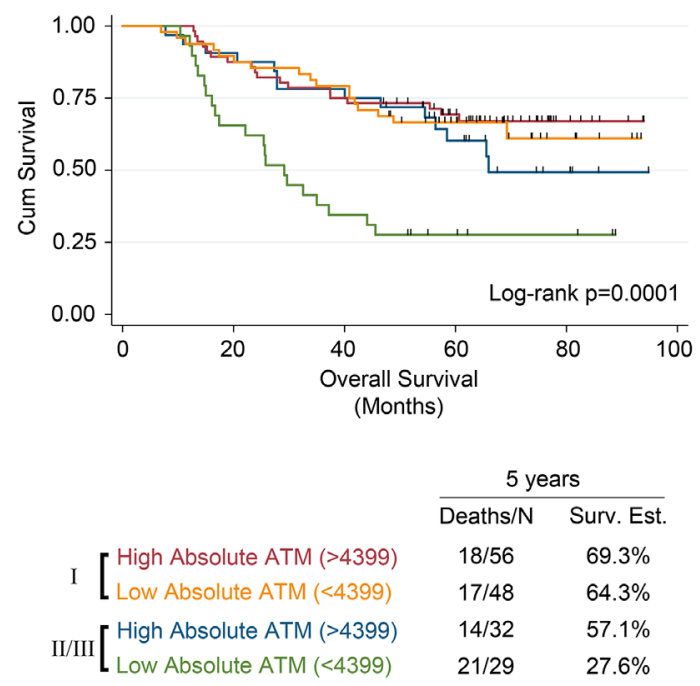

D
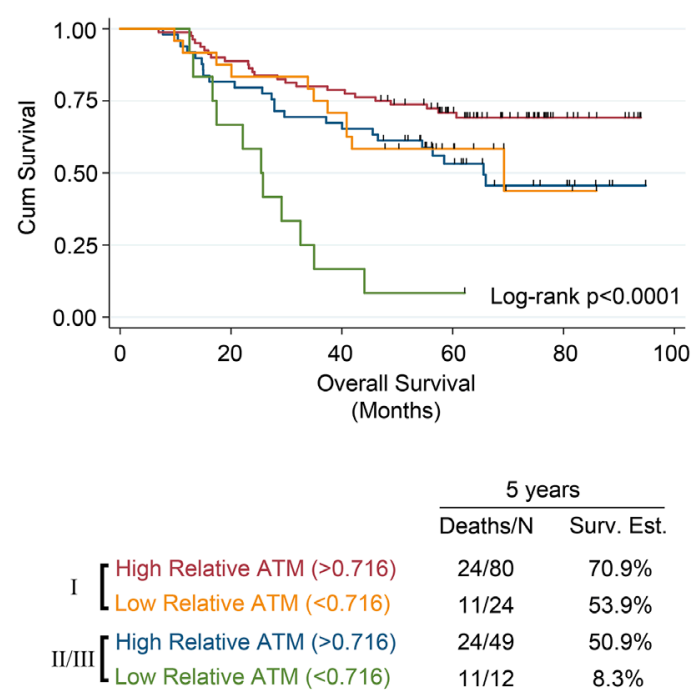

Figure 3: Univariate survival analysis in NSCLC patients stratified by stage and based on ATM expression measured using malignant cell-specific ATM and ATM expression index scores. Kaplan-Meier analysis and 5-year survival estimates, stratified into stage I or stages II/III, for (A) disease free survival and (B) overall survival in NSCLC patients with low or high malignant cell specific ATM expression scores. Kaplan-Meier analysis and 5-year survival estimates, stratified into stage I or stages II/III, for (C) disease free survival and (D) overall survival in NSCLC patients with low or high ATM expression index scores. All Kaplan-Meier curves include the 5 -year event rate expressed at the Events/ $\mathrm{N}$, where $\mathrm{N}$ is the number at risk. 
Table 2: Cox proportional hazards regression models in early stage lung cancer cohort - comparing low and high ATM expression in stage II/III patients

5 Year Disease Free Survival

5 Year Overall Survival

Factor

Hazard Ratio (95\% C.I.) p-Value Hazard Ratio (95\% C.I.) $p$-Value

\begin{tabular}{lcccc}
\hline Malignant Cell-Specific ATM $(>4399 /<4399)$ & $1.88(0.96-3.69)$ & 0.068 & $2.79(1.38-5.66)$ & $0.004^{\mathrm{a}}$ \\
Adjuvant Treatment (No/Yes) & $0.65(0.33-1.29)$ & 0.222 & $0.78(0.39-1.55)$ & 0.476 \\
Adenocarcinoma (No/Yes) & $0.40(0.12-1.31)$ & 0.128 & $0.59(0.17-2.02)$ & 0.396 \\
BAC (No/Yes) & $0.27(0.05-1.45)$ & 0.127 & $0.17(0.02-1.57)$ & 0.117 \\
Squamous Cell (No/Yes) & $0.31(0.10-0.98)$ & $0.047^{\mathrm{a}}$ & $0.53(0.16-1.80)$ & 0.312 \\
Gender (Female/Male) & $1.27(0.62-2.61)$ & 0.514 & $1.82(0.87-3.82)$ & 0.113 \\
Age (Continuous) & $1.00(0.96-1.03)$ & 0.873 & $1.02(0.98-1.06)$ & 0.250 \\
ATM Expression Index (>0.716/<0.716) & $4.75(2.02-11.17)$ & $<0.001^{\mathrm{a}}$ & $5.09(2.07-11.17)$ & $<0.001^{\mathrm{a}}$ \\
Adjuvant Treatment (No/Yes) & $0.69(0.35-1.34)$ & 0.272 & $0.87(0.44-1.71)$ & 0.680 \\
Adenocarcinoma (No/Yes) & $0.46(0.14-1.49)$ & 0.196 & $0.73(0.22-2.46)$ & 0.609 \\
BAC (No/Yes) & $0.32(0.06-1.70)$ & 0.182 & $0.20(0.02-1.81)$ & 0.151 \\
Squamous Cell (No/Yes) & $0.44(0.14-1.42)$ & 0.171 & $0.90(0.26-3.06)$ & 0.863 \\
Gender (Female/Male) & $1.21(0.61-2.42)$ & 0.581 & $1.81(0.88-3.74)$ & 0.109 \\
Age (Continuous) & $1.01(0.97-1.05)$ & 0.627 & $1.04(1.00-1.08)$ & 0.057 \\
\hline
\end{tabular}

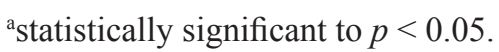

early resectable NSCLC lend support to the hypothesis that ATM loss is an important event in NSCLC carcinogenesis.

By using the digital image analysis capability of the HistoRx AQUA ${ }^{\circledR}$ platform, we were able to quantify ATM protein levels and to define the specific localization of ATM expression within the tumour. Using the ratio of ATM expression in the malignant component compared to the stromal elements of the tumor (the ATM-EI), we defined a cohort of $22 \%$ of patients whose NSCLC tumors could be described as being ATM-deficient. Interestingly, there was no difference in the incidence of relative ATM deficiency between stage I and stage II/III patients. This methodology for determining ATM-deficiency proved more stringent than standard histopathology staining, defining $22 \%$ of patients in our cohort as ATM-deficient, compared to $40 \%$ deficiency in a similar-sized cohort, recently reported by Villaruz et al. [19]. Similarly, quantitative IHC of ATM in breast cancer identified a smaller subgroup of ATMdeficient patients [26] than does standard histopathology [27], however in both of these studies ATM-loss was associated with poor DFS.
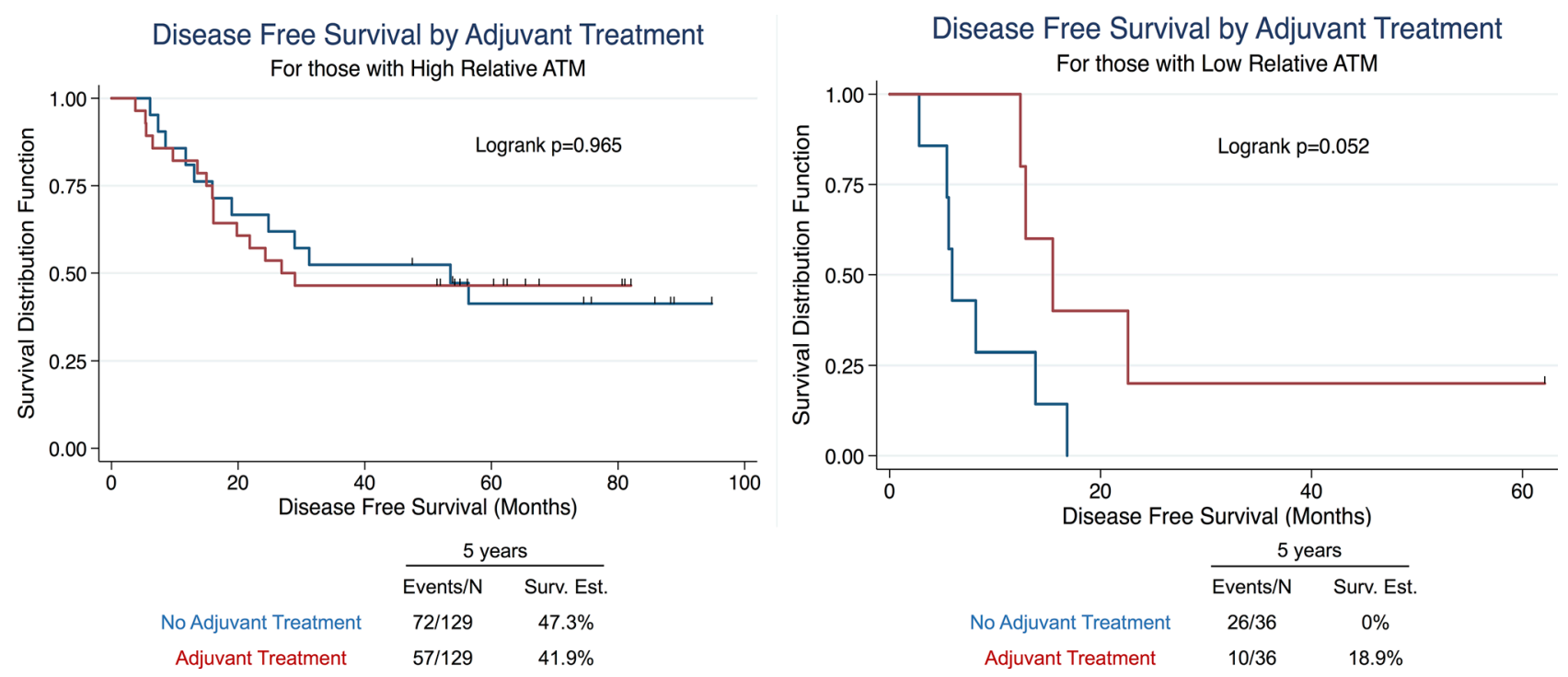

Figure 4: Univariate survival analysis in NSCLC patients stratified by adjuvant treatment in high ATM-EI and low ATM-EI groups. Kaplan-Meier analysis and 5-year disease free survival estimates, stratified into Adjuvant Treatment or No Adjuvant Treatment for (A) high ATM expression index, and (B) low ATM expression index scores. All Kaplan-Meier curves include the 5-year event rate expressed at the Events/ $\mathrm{N}$, where $\mathrm{N}$ is the number at risk. 
By using the accompanying outcome data in the Glans-Look lung cancer database, we confirm that measuring absolute ATM level in samples does not significantly predict survival in early-resected NSCLC, as was previously reported [19], however we show that when ATM deficiency is defined as a ratio of tumour to stromal expression below 0.716, ATM loss is an independent predictor of both 5-year DFS and 5-year OS. This analysis is arguably more biologically relevant, as the genomic instability induced by ATM loss specifically in the cancerous cells could drive progression of the tumor. Certainly, variable expression of biomarkers including ATM in cancer-associated stromal tissues have been observed [26, 28], and higher levels are associated with aggressive characteristics of breast cancer [26]. While stromal ATM expression in our samples did not predict outcome (not shown), the ATM-EI value does consider the stromal compartment in the calculation.

The fact that relative ATM deficiency is seen in a proportion of stage I patients suggests that reduced ATM expression is an early event in NSCLC carcinogenesis and supports the hypothesis [29] that tumour progression is associated with a disruption in the ATM-CHK2-p53 pathway in many human cancers, including lung cancer. Intriguingly, there was no clear association between ATM deficiency and smoking, perhaps indicating that disruption in the ATM-CHK2-p53 pathway is one of several mechanisms by which lung carcinogenesis can occur. An association between genomic instability and increased immunogenicity has been proposed. Recent data suggest that immune-modulating treatments have a role to play in NSCLC where efficacy is seen regardless of smoking status [30, 31]. Our data therefore lends a mechanistic basis for this observation.

Perhaps more important from a patient perspective is our observation that adjuvant treatment positively influences the 5-year DFS of the low ATM-EI group, suggesting that ATM-deficiency may increase sensitivity to certain chemotherapies. This is consistent with recent reports and our own data (not shown) that have shown increased platin-induced cytotoxicity in vitro in ATM- and DDR-deficient cell lines [32, 33]. Additionally, ATM-loss in breast cancer If this is the case, our data suggests that while patients with early stage ATM-proficient NSCLC receive little-to-no advantage from adjuvant therapy, those with ATM-deficient tumours could benefit greatly under current standard treatment regimens, or even low dose therapy.

Our study has inherent limitations. It did not set out to investigate the mechanisms underlying the loss of ATM expression in NSCLC. In addition to the many ATM mutations described in the literature, several other mechanisms for altering or silencing ATM have been delineated [34]. Furthermore, a simple measure of ATM protein expression by IHC cannot establish the functional capability of ATM within the tumour. It is possible that in some tumours that are shown to be ATM deficient by IHC,
ATM functionality is still preserved, while in other cases ample ATM expression by IHC could be detecting nonfunctional, truncated protein. Other studies suggest that p53 loss may further influence the role of ATM deficiency on cellular phenotype including chemosensitivity [23]. Clearly, this represents a therapeutically exploitable deficit and may help target the use of non-specific DNA damaging agents such as ionizing radiation, cytotoxic drugs and more targeted agents such as PARP inhibitors in future treatment algorithms for advanced NSCLC.

In summary, we report that loss of ATM expression, as denoted by a low ATM-EI within the malignant component of a tumour, can be detected in a substantial proportion of resected NSCLCs. Such loss of ATM is seen in many stage I tumours, implying that this is a relatively early event in NSCLC tumourigenesis. Our analysis also suggests that a low ATM-EI confers a poor prognosis in terms of both DFS and OS on patients despite resection. The implications of ATM loss may also have a predictive utility since several therapeutic modalities used in NSCLC may exploit this deficiency. Investigations that determine the underlying mechanisms of ATM loss may reveal different molecular pathways of carcinogenesis that ultimately lead to NSCLC. Meanwhile, further clinical studies on the association between this therapeutically important protein and outcome and response, in early and advanced stage NSCLC, will be important to improve the targeting and efficacy of existing treatment modalities.

\section{MATERIALS AND METHODS}

\section{Case selection and clinical data collection}

This study was approved by the University of Calgary Conjoint Faculties Research Ethics Board, in accordance with the Tri-Council Policy Statement on Research with Human Subjects. Clinical data was collected retrospectively through chart review of NSCLC patients diagnosed at the Tom Baker Cancer Centre from 2003 to 2006 and entered into the Glans-Look Lung Cancer Database as previously described [35]. Tumour resection type was divided into wedge resection, lobectomy and more extensive surgery (bi-lobectomy, hemi-pneumonectomy, or a pneumonectomy). Adjuvant treatment included patients who received adjuvant chemotherapy alone, adjuvant chemoradiotherapy, and those who received neoadjuvant radiotherapy.

\section{Tissue microarray generation}

All archived formalin-fixed paraffin-embedded (FFPE) resected NSCLC tumour samples were retrieved from Calgary Laboratory Services and reviewed by a pathologist (AMM). Tissue microarrays where constructed as previously described [35]. Normal lung tissue specimens $(n=21)$, normal tonsil tissue, an ATM-deficient 
human lymphoblastoid cell line (L3) and an age matched ATM-proficient human lymphoblastoid cell line (BT/ C3ABR) were also included as controls [23].

\section{Four-colour fluorescence immunohistochemistry}

Sections ( $4 \mu \mathrm{m}$-thick) from each TMA block were processed and stained as previously described $[35,36]$. Slides were processed using a Dako Autostainer and incubated for 60 minutes with a cocktail of three primary antibodies, including: anti-pan-cytokeratin to identify tumour cells, anti-vimentin to identify stromal cells, and anti-ATM (described in Supplementary Table 1). The antiATM antibody was previously validated and characterized [19]. Protein expression was visualized with consecutive applications of secondary antibodies and fluors as previously described [35].

\section{Automated image acquisition and analysis}

Automated image acquisition was performed using a HistoRx PM-2000 ${ }^{\mathrm{TM}}$, previously been described in detail [22]. Briefly, high resolution monochromatic 8-bit digital images were obtained for every histospot on the TMAs using filters specific for DAPI, FITC to define cytokeratin positive NSCLC cells, Cy3 to define vimentin positive non-malignant stromal cells, and Cy5 to define the target biomarker ATM. Images were then analyzed using the AQUAnalysis ${ }^{\circledR}$ program, version 2.2.1.7 as previously described [36].

\section{Assessment of ATM deficiency}

The average intensity of target ATM signal in the masked areas was tabulated and used to generate malignant cell-specific, and non-malignant tumourassociated stromal cell-specific AQUA scores, which reflect the average signal intensity per malignant area, and stromal area, respectively. The ATM expression score was defined as the minimum ATM malignant cellspecific AQUA score from the triplicate cores for each patient sample. The ATM expression index was defined as the minimum ratio, from the triplicate cores for each patient sample, of the malignant cell-specific AQUA score as compared with the non-malignant tumour-associated stromal cell-specific AQUA score.

\section{Statistical analysis}

Cut-points to identify two groups from the minimum ATM expression score and the minimum ATM expression index levels were found using a method based on the logrank test statistic [24]. Differences in demographic and clinicopathological characteristics were assessed across the two groups using chi square tests for categorical data and $t$-tests for continuous data. Differences in the survival were compared using the log-rank test for low versus high ATM expression index groups and ATM expression groups as well as the stage subgroups. Cox proportional hazards regression was used to assess the prognostic effect of both ATM expression index and tumour ATM. Demographic and clinicopathological factors were included in the multivariable Cox proportional hazards models using an epidemiological framework. Variables were first assessed as potential modifiers and in the absence of the modification variables were assessed for their potential effect as confounders of the investigation under consideration. Proportional hazards assumptions were examined and confirmed by log-negative log KaplanMeier curves and by inspecting interaction terms of log (survival time) by the covariates of interest. Five-year disease free and overall survival estimates were calculating using the Kaplan-Meier method. All statistical analyses were conducted using SAS/STAT software (Version 9.2, SAS Institute Inc, Cary, NC, USA).

\section{ACKNOWLEDGMENTS AND FUNDING}

This work was made possible by an operating grant awarded to DGB and SLM from Alberta Innovates - Health Solution and donations to DGB through the University of Calgary to fund the creation and development of the Glans-Look Lung Cancer database.

\section{CONFLICTS OF INTEREST}

The authors declare that no conflicts of interest exists.

\section{REFERENCES}

1. Lawrence MS, Stojanov P, Polak P, Kryukov GV, Cibulskis K, Sivachenko A, Carter SL, Stewart C, Mermel CH, Roberts SA, Kiezun A, Hammerman PS, McKenna A, et al. Mutational heterogeneity in cancer and the search for new cancer-associated genes. Nature. 2013; 499:214-8

2. Alexandrov LB, Nik-Zainal S, Wedge DC, Aparicio SA, Behjati S, Biankin AV, Bignell GR, Bolli N, Borg A, Borresen-Dale AL, Boyault S, Burkhardt B, Butler AP, et al. Signatures of mutational processes in human cancer. Nature. 2013; 500:415-21.

3. Thu KL, Vucic EA, Chari R, Zhang W, Lockwood WW, English JC, Fu R, Wang P, Feng Z, MacAulay CE, Gazdar AF, Lam S, Lam WL. Lung adenocarcinoma of never smokers and smokers harbor differential regions of genetic alteration and exhibit different levels of genomic instability. PLoS One. 2012; 7:e33003.

4. Bedard PL, Hansen AR, Ratain MJ, Siu LL. Tumour heterogeneity in the clinic. Nature. 2013; 501:355-64.

5. Scagliotti GV, De Marinis F, Rinaldi M, Crino L, Gridelli C, Ricci S, Matano E, Boni C, Marangolo M, 
Failla G, Altavilla G, Adamo V, Ceribelli A, et al. Phase III randomized trial comparing three platinum-based doublets in advanced non-small-cell lung cancer. J Clin Oncol. 2002; 20:4285-91.

6. Pikor L, Thu K, Vucic E, Lam W. The detection and implication of genome instability in cancer. Cancer Metastasis Rev. 2013.

7. Loeb LA. A mutator phenotype in cancer. Cancer Res. 2001; 61:3230-9.

8. Loeb LA. Human cancers express mutator phenotypes: origin, consequences and targeting. Nat Rev Cancer. 2011; 11:450-7.

9. Abraham RT. The ATM-related kinase, hSMG-1, bridges genome and RNA surveillance pathways. DNA Repair (Amst). 2004; 3:919-25.

10. Abraham RT. PI 3-kinase related kinases: 'big' players in stress-induced signaling pathways. DNA Repair (Amst). $2004 ; 3: 883-7$.

11. Haince JF, Kozlov S, Dawson VL, Dawson TM, Hendzel MJ, Lavin MF, Poirier GG. Ataxia telangiectasia mutated (ATM) signaling network is modulated by a novel poly(ADPribose)-dependent pathway in the early response to DNAdamaging agents. J Biol Chem. 2007; 282:16441-53.

12. Kang B, Guo RF, Tan XH, Zhao M, Tang ZB, Lu YY. Expression status of ataxia-telangiectasia-mutated gene correlated with prognosis in advanced gastric cancer. Mutat Res. 2008; 638:17-25.

13. Kim NG, Choi YR, Baek MJ, Kim YH, Kang H, Kim NK, Min JS, Kim H. Frameshift mutations at coding mononucleotide repeats of the hRAD50 gene in gastrointestinal carcinomas with microsatellite instability. Cancer Res. 2001; 61:36-8.

14. Stankovic T, Weber P, Stewart G, Bedenham T, Murray J, Byrd PJ, Moss PA, Taylor AM. Inactivation of ataxia telangiectasia mutated gene in B-cell chronic lymphocytic leukaemia [see comments]. Lancet. 1999; 353:26-9.

15. Schaffner C, Stilgenbauer S, Rappold GA, Dohner H, Lichter P. Somatic ATM mutations indicate a pathogenic role of ATM in B-cell chronic lymphocytic leukemia. Blood. 1999; 94:748-53.

16. Greenman C, Stephens P, Smith R, Dalgliesh GL, Hunter C, Bignell G, Davies H, Teague J, Butler A, Stevens C, Edkins S, O'Meara S, Vastrik I, et al. Patterns of somatic mutation in human cancer genomes. Nature. 2007; 446:153-8.

17. Schneider J, Illig $\mathrm{T}$, Rosenberger A, Bickeboller $\mathrm{H}$, Wichmann HE. Detection of ATM gene mutations in young lung cancer patients: a population-based control study. Arch Med Res. 2008; 39:226-31.

18. Weber AM, Drobnitzky N, Devery AM, Bokobza SM, Adams RA, Maughan TS, Ryan AJ. Phenotypic consequences of somatic mutations in the ataxia-telangiectasia mutated gene in non-small cell lung cancer. Oncotarget. 2016; 7:60807-60822. doi: 10.18632/oncotarget.11845.
19. Villaruz LC, Jones H, Dacic S, Abberbock S, Kurland BF, Stabile LP, Siegfried JM, Conrads TP, Smith NR, O'Connor MJ, Pierce AJ, Bakkenist CJ. ATM protein is deficient in over $40 \%$ of lung adenocarcinomas. Oncotarget. 2016; 7:57714-57725. doi: 10.18632/oncotarget.9757.

20. Lavin MF, Birrell G, Chen P, Kozlov S, Scott S, Gueven N. ATM signaling and genomic stability in response to DNA damage. Mutat Res. 2005; 569:123-32.

21. McShane LM, Altman DG, Sauerbrei W, Taube SE, Gion M, Clark GM, Statistics Subcommittee of NCIEWGoCD. REporting recommendations for tumor MARKer prognostic studies (REMARK). Breast Cancer Res Treat. 2006; 100:229-35.

22. Camp RL, Chung GG, Rimm DL. Automated subcellular localization and quantification of protein expression in tissue microarrays. Nat Med. 2002; 8:1323-7.

23. Williamson CT, Kubota E, Hamill JD, Klimowicz A, Ye R, Muzik H, Dean M, Tu L, Gilley D, Magliocco AM, McKay BC, Bebb DG, Lees-Miller SP. Enhanced cytotoxicity of PARP inhibition in mantle cell lymphoma harbouring mutations in both ATM and p53. EMBO Mol Med. 2012; 4:515-27.

24. Contal C OQJ. An application of changepoint methods in studying the effect of age on survival in breast cancer. Comput Stat Data Analysis. 1999; 30:253-70.

25. Hanahan D, Weinberg RA. Hallmarks of cancer: the next generation. Cell. 2011; 144:646-74.

26. Feng X, Li H, Dean M, Wilson HE, Kornaga E, Enwere EK, Tang P, Paterson A, Lees-Miller SP, Magliocco AM, Bebb G. Low ATM protein expression in malignant tumor as well as cancer-associated stroma are independent prognostic factors in a retrospective study of early-stage hormonenegative breast cancer. Breast Cancer Res. 2015; 17:65.

27. Suh KJ, Ryu HS, Lee KH, Kim H, Min A, Kim TY, Yang Y, Moon HG, Han SW, Oh DY, Han W, Park IA, Noh DY, et al. Loss of ataxia-telangiectasia-mutated protein expression correlates with poor prognosis but benefits from anthracycline-containing adjuvant chemotherapy in breast cancer. Breast Cancer Res Treat. 2016; 158:233-41.

28. Peng Q, Zhao L, Hou Y, Sun Y, Wang L, Luo H, Peng H, Liu M. Biological characteristics and genetic heterogeneity between carcinoma-associated fibroblasts and their paired normal fibroblasts in human breast cancer. PLoS One. 2013; 8:e60321.

29. Bartkova J, Horejsi Z, Koed K, Kramer A, Tort F, Zieger K, Guldberg P, Sehested M, Nesland JM, Lukas C, Orntoft T, Lukas J, Bartek J. DNA damage response as a candidate anti-cancer barrier in early human tumorigenesis. Nature. 2005; 434:864-70.

30. Scoria JC, Cruz C, Bahleda R, Delord JP, Horn L, Herbst RS, Spigel D, Mokatrin A, Fine G, Gettinger S. Clinical activity, safety and biomarkers of PD-L1 blockade in non-small cell lung cancer (NSCLC): Additional 
analyses from a clinical study of the engineered antibody MPDL3280A (anti-PDL1). European Cancer Congress. 2013.

31. Brahmer JR, Tykodi SS, Chow LQ, Hwu WJ, Topalian SL, Hwu P, Drake CG, Camacho LH, Kauh J, Odunsi K, Pitot HC, Hamid O, Bhatia S, et al. Safety and activity of anti-PD-L1 antibody in patients with advanced cancer. N Engl J Med. 2012; 366:2455-65.

32. Sears CR, Cooney SA, Chin-Sinex H, Mendonca MS, Turchi JJ. DNA damage response (DDR) pathway engagement in cisplatin radiosensitization of non-small cell lung cancer. DNA Repair (Amst). 2016; 40:35-46.

33. Lopez-Martinez D, Liang CC, Cohn MA. Cellular response to DNA interstrand crosslinks: the Fanconi anemia pathway. Cell Mol Life Sci. 2016; 73:3097-114.

34. Bai AH, Tong JH, To KF, Chan MW, Man EP, Lo KW, Lee JF, Sung JJ, Leung WK. Promoter hypermethylation of tumor-related genes in the progression of colorectal neoplasia. Int J Cancer. 2004; 112:846-53.

35. Otsuka S, Klimowicz AC, Kopciuk K, Petrillo SK, Konno M, Hao D, Muzik H, Stolte E, Boland W, Morris D,
Magliocco AM, Bebb DG. CXCR4 overexpression is associated with poor outcome in females diagnosed with stage IV non-small cell lung cancer. J Thorac Oncol. 2011; 6:1169-78.

36. Brockton NT, Klimowicz AC, Bose P, Petrillo SK, Konno M, Rudmik L, Dean M, Nakoneshny SC, Matthews TW, Chandarana S, Lau HY, Magliocco AM, Dort JC. High stromal carbonic anhydrase IX expression is associated with nodal metastasis and decreased survival in patients with surgically-treated oral cavity squamous cell carcinoma. Oral oncology. 2012; 48:615-22. 